Rasse "hervorragende geistige Eigenschaften, höchstentwickelte Sprachen und weltbeherrschende Kultur" zuzuschreiben ${ }^{1}$ ). Davon kann höchstens in der Neuzeit, nicht aber in früheren Zeiten die Rede sein; 'höchstentwickelte' Sprachen ist zudem ein sehr vager Begriff. S. 16 werden die Rätier als Thraker, und die Etrusker als Indogermanen angesprochen; S. 18 wird die nähere Verwandtschaft des Lateinischen mit den nordischgermanischen Sprachen mit ganz willkürlichen gewählten Gleichungen zu erweisen gesucht. DaB S. 26 auch die Runenschrift bereits als indogermanischer Kulturbesitz reklamiert wird, dürfte wohl das Stärkste sein, was sich Verf. an historischen Unmöglichkeiten leistet. Zwei Nachträge befassen sich speziell mit der Urheimat der Germanen und den Ansichten des Kunsthistorikers G. Steinhausen sowie des schwedischen Gelehrten Axel Kock hierüber.

Bespr.: Zschr. f. Ethnol. 1907, 1008f. von P. Ehrenreich.

Berlin.

S. Feist.

\title{
II. Arisch.
}

\section{A. Indo-Iranisch.}

1. Scherman L. Orientalische Bibliographie (begründet von August Müller). 21. Bd. (für 1907). Drei Hefte in einem Bande. Berlin, Reuther u. Reichard 1909. IV u. $400 \mathrm{~S}$. 80. Subskr.-Pr. $12 \mathrm{M}$.

IV. Indogermanen. 1. Allgemeines (S. 150-154, Nr. 2926-3034).

2. Indien (S. 154-200, Nr. 3035-4046). Rezensionen zu IV, 1-2 (S. 201-205).

3. Iran (S. 205-214, Nr. 4047-4262). Rezensionen zu IV, 3 ff. (S. 225-227).

2. Ciardi-Dupré G., Aucher P. G., Bonelli L., Ballini A. Lingua e letteratura armena. Lingue e letterature indo-eraniche. Riv. degli stud. or. 1, $505-572$.

Eine Besprechung der auf armenischem, indo-iranischem, indischem und iranischem Gebiete erschienenen Publikationen, von denen an dieser Stelle nur Indo-Iranisch und Indisch in Betracht kommen.

Glottologie S. 505-514: Indo-iranische Sprachen: S. 507-512, A. Indisch und Iranisch, S. $507 \mathrm{f}$ - - B. Indisch, S. 508-511.

Altindisch S. 538-572: Bibliographie, Hss.-Kataloge, Lexikographie, Grammatik, Rhetorik, Paläographie, S. 538-541. - Veda, Brāhmana, Sūtra, Upanișad, S. 541-545. - Philosophie, S. 545-548. - Buddhismus, S. 548-552. - Jainismus, S. 552-554. - Geschichte, Geographie, Ethnographie, S. 554-558. - Sanskritliteratur, S. 559-561. - Jurisprudenz, S. 561 f. - Medizin, Astronomie, Alchimie, S. 562 f. - Epigraphik, Numismatik, Archäologie, Kunst, S. 563-567. - Pāli und moderne indische Dialekte, S. 567-569. - Biographisches, S. 569 f. - Varia, S. 570-572.

1) Ganz entgegengesetzt urteilt z. B. Gustav Retzins im Journal of the Royal Anthropological Institute 39, $299 \mathrm{ff}$., der als Charakteristikum der dolichokephalen nordischen Rasse eine bedeutende geistige Regsamkeit, aber keine Ausdauer bei ihren Unternehmungen ansieht. Deswegen werde sie überall von der beharrlicheren brachykephalen Rasse Mitteleuropas mehr und mehr zurückgedrängt. Man sieht, wie der hervorragende nordische Anthropologe die Rassetheorien der Chamberlain, Gobineau, Woltmann usw. zuschanden macht. 
3. Meillet A. Le dieu indo-iranien Mitra. JA., Sér. X, t. 10, 143-159.

Präzisierung und Rektifizierung der bis jetzt herrschenden Anschauung, daß Mitra ein Licht-, spez. ein Sonnengott ist. "L'indo-iranien Mitra est le contrat, la puissance mystique du contrat, et une personne; et les trois notions s'interchangent constamment" ... "La doctrine du mithriacisme romain ne possédait plus ... que peu de traits communs avec la notion simple, mais si conforme en type religieuse de l'époque indoeuropéenne : la puissance immanente du contrat-dieu, omniscient, surveillant tout, ayant pour cil le soleil, voyant tout, allant partout, soutenant l'ordre du monde, et châtiant avec une force redoutable les infractions commises par les hommes et par les dieux: ce n'est pas un phénomène naturel, c'est un phénomène social divinisé.

\section{B. Indisch.}

\section{Allgemeines. Geschichte.}

1. Oldenberg H. Indologie. Internat. Wochenschrift 1907. S. 635-644. Hinweis auf Inhalt und Ziele der Indologie, auf ihre Wichtigkeit für linguistische Aufgaben (Lautverschiebung, Vokalisationssystem, Geschichte der Laute, Worte, Wortsysteme, Bedeutungsnuancen; Einfluß der indischen Grammatik), auf den Wert der indischen literarischen Überlieferung für Religion, Philosophie und Poesie (Erzählung, Märchen, Drama). Charakteristik der Anteile der einzelnen Völker an der Erforschung Indiens: Engländer, Russen, neuerdings auch Franzosen und Japaner betreiben infolge ihrer unmittelbaren Beziehungen zu Indien mehr das Aufsuchen und Sammeln neuen Materials, während der Deutsche vorzüglich die kritische Bearbeitung des Stoffes unternimmt und die so gewonnenen Resultate in größere Zusammenhänge einordnet. Das Studium des indischen Altertums verschafft außerdem Übung und Erfahrung im Lösen entsprechender Aufgaben auch auf anderen Gebieten und gibt Anregung zur Entwicklung und Verfeinerung immer weiter tragender Untersuchungsmethoden.

2. Klemm K. Inder (bis zur Gegenwart). Jahresberichte der Geschichtswissensch. (für 1905). $28 \mathrm{I}, 97-124$.

Zusammenstellung und Charakterisierung der 1905 erschienenen Publikationen über Vorgeschichte, Ethnographie, Ären, Geschichte, historische Geographie, Archäologie, Inschriftenkunde, Grapbik, Religionsgeschichte, Philosophie, Staat, Recht und Sitte, Sprachwissenschaft, Literaturgeschichte, Heilkunde, Grenzgebiete (Nepāl, Ladākh), Tibet, Kaschgar; Hinterindien, Indo-China, Malaiische Halbinsel, Niederländisch-Indien.

3. Gjellerup K. Indisches. Preub. Jahrb. 129, 335-342.

Bespricht: 1. Hermann Oldenberg, Vedaforschung. Stuttgart 1905.

- 2. Paul Deussen, Vier philosophische Texte des Mahabharatam. Leipzig 1906. - 3. Richard Garbe, Die Bhagavadgitã. Leipzig 1905. - 4. A. Paul, Krishnas Weltengang. München 1905. - 5. Julius Dutoit, Das Leben des Buddha. Leipzig 1906. - 6. Julius Dutoit, Jatakam. Leizpig 1906.

4. Oldenberg, H. Geschichtschreibung im alten Indien. Dt. Rdsch. 1907. 130, 362-379.

Versuch einer Beantwortung der Frage: Wie weit haben die Inder die Aufgaben histor. Denkens, histor. Darstellung verstanden und gelöst? Eine Art Anfänge ind. Geschichtschreibung bilden die Puranas, Sammel- 
surien von Mythen, Legenden, wirklichen Ereignissen, Vorschriften usw. Unabhängig von ihnen entwickelten sich die Erzählungen von Buddha und seinen Anhängern sowie die romanhaften Geschichten berïhmter Könige. In den Vordergrund seiner Betrachtung rïckt 0 . den Mahavansa (d. i. grobe Chronik) und den Radschatarangini (d. i. Strom der Könige). Der erstere, verfaßit von dem buddhist. Mönche Mahanama in Ceylon gegen $500 \mathrm{n}$. Chr, zeigt eine Art Verwandtschaft mit den Mönchchroniken des Mittelalters, doch ist ex flacher, plumper, phantastischer und unwahrer. Der Radschatar., eine histor. Dichtung, verfaßt von Kalhana um 1150 n. Chr. in Kaschmir, ist voll chronologischer Verwirrung, die erst für die spätere Zeit größerer Zuverlässigkeit weicht. Bei Schilderung der von ihm miterlebten Ereignisse sucht er Charakter und Absicht der handelnden Personen zu verstehen, ohne es jedoch hierin zu gröberer Feinheit der Auffassung zu bringen. Beide Werke bleiben zweifellos diesseits des Punktes stehen, wo die Geschichten von Königen und merkwürdigen Taten sich in wirkliche Geschichte verwandeln. Auf jeden Fall aber repräsentiert der Radschatar. den Höhepunkt ind. Geschichtschreibung. 0 . gelangt schließlich zu folgendem Resultat: Allerdings haben die Inder eine Geschichtschreibung besessen, aber nur als Erzählung einzelner Ereignisse. 'Zum Durchdenken der geschichtlichen Zusammenhänge, des Wirkens der Kausalitäten haben sie sich kaum erhoben. . . Sodann wäre die ungewöhnliche Lückenhaftigkeit und Verwirrtheit des Wissens namentlich von den älteren Zeiten hervorzuheben', sowie die Einflüsse von Phantasie, Wunderglauben, Religion und Poesie. Als Ursachen hiervon möchte O. ansehen den späten und sparsamen Gebrauch der Schrift, die Vergänglichkeit der Handschriften, die fortwährenden politischen Umwälzungen, den despotischen Charakter des ind. Staatswesens, den südlichen Geschmack an Buntem, Grellem und Ungeheuerlichem und in Verbindung damit grobe Leichtgläubigkeit und stark zurückgeschobenen Wahrheitssinn.

5. Widney J. P. Race life of the Aryan peoples. Vol. 1: The old world. 2: The new world. New York, Funk and Wagnalls 1907. VII, 347; VII, 359 S. 4 Doll.

Bespr.: Athenaeum 1907. 2, 484f.; Woodruff, C. E., N. Y. Times SR 1907. $497 \mathrm{f}$; Outlook 86, 973; Independent 63, 1375 .

6. Dutt R. C. A brief history of ancient and modern India, according to the syllabus prescribed by the Calculta University. Calcutta, S. K. Láhiri and Co. London, Paul 1907. S. 211, 1 K. (ill.). 1 R. \& a.; 2 s.

7. Büttner 0. Indien und das Kastenwesen. Hochland 1906/07. 4, I, 737-744.

Geschichtliche Entwicklung. Aus den 4 alten Kasten entstanden im Laufe der Jahrhunderte unzählige Unterkasten in Gestalt von Korporationseinheiten, die in der Regel durch einen gemeinsamen Beruf zusammengehalten werden. So viel Kasten, so viel Gegensätze auch! Die trennenden Momente bilden hierbei Rasse und Beruf, Stammesverschiedenheit und Wohnsitz, Ehegesetze und sonstige Sondersitten. Doch wäre die Kastengliederung nicht so bedeutend geworden, hätte sie nicht eine Stütze an der Religion gehabt, denn das Kastenwesen wird als Ausfub des göttlichen Willens hingestellt, gegen den sich aufzulehnen sündhaft ist. Diese soziale Spaltung hat einerseits den Klassenhaß gewaltig geschärft, andererseits das Gefühl nationaler Zusammengehörigkeit ertötet. 
8. Jackson A. M. T. Note on the history of the caste system. J. a. Proc. ASB. 3, 509-515.

Versuch einer Erklärung der unendlich großen Menge moderner Kasten aus den ursprünglichen 4 Kasten der Hindu-Gesetzbücher. In den früheren Zeiten der politischen Selbständigkeit der Hindus bestand die ganze indische Lebensgemeinschaft aus hierarchisch organisierten Kasten mit Brahmanen und einem Könige an der Spitze, welch' letzterer die Aufgabe hatte, jede Kaste innerhalb der Grenzen der ihr zukommenden Funktionen zu halten und eine Vermischung der Kasten (Varnasankara) zu verhindern. Hieraus baben sich dann im Laufe der Zeit zahlreiche Kastengebräuche entwickelt, die schlieblich zu weiteren (Unter-)Kastenbildungen geführt haben dürften, deren Namen vielfach geographischen Bezeichnungen (Länder- und Städtenamen) entlehnt sind. Wenn natürlich auch nicht alle modernen Kasten auf die Kasten der alten Hindu-Königreiche zurückzuführen sind, so wird doch die Jurisdiktion der Hindukönige ein malgebender Faktor in der Entstehung des gesamten Kastensystems gewesen sein.

9. Cajus J. Au pays des castes. Les Brahmanes. Anthropos. 2, 35-39.

I. Chronologisch geordnete Bibliographie.

10. Vaidya C. V. Epic India or India as described in the Mahabharata and the Ramayana. Bombay 1907. 516 S.; $1 \mathrm{~K}$. (Leipzig, Harrassowitz) 7,50 M.

11. Smith V. A. The Śakas in Northern India. ZDMG. 61, 403-421.

Stellungnahme Smiths betreffs der Beziehungen zwischen Sakas, Indo-Parthern und Kushāns (als ein Supplement zu seinem Essay 'The Indo-Parthian Dynasties', und mit Bezugnahme auf Fleets Anschauungen über diesen Gegenstand, veröffentlicht im Journal of the RAS.). Die Sakas in Nordindien sind für Sm. eine feststehende Tatsache; ihr Einfall in das Fünfstromland dürfte im 2. Jahrh. v. Chr. erfolgt sein. In der 2. Hälfte des 1. Jahrh. n. Ghr. von den stammverwandten Yüe-Ghi (bekannt in der Sanskritliteratur als Tusāras, Tukhäras oder Turușkas) unterworfen, gingen sie allmählich in diesen unter. Jedenfalls werden Maues und seine Nachfolger Sakas gewesen sein.

12. Har Bilas Sarda. Hindu superiority. Ajmer (London, Probsthain) 1907. 454 S. (ill.). 12 s. 6 d.

Inhalt: Constitution. Hindu colonization. Literature. Philosophy. Science. Arts. Commerce. Religion.

13. Dyroff A. Eine indische Ästhetik. Arch. f. Gesch. der Philos. 1905. $18,113-134$.

Eine Darlegung des Wesens ind. Ästhetik in Form eines Hinweises auf Inhalt und Eigenart einer von Jacobi in ZDMG. 56 und 57 übersetzten ind. Poetik, deren Vorschriften sich in der Theorie aber auch auf jede andere Kunst übertragen lassen. Zunächst einige Bemerkungen über die vorausliegende Zeit: Prinzip von den 10 Fehlern und 10 Vorzügen eines Werkes; Schönheit der sprachlichen Form und des gedanklichen Ausdrucks. Theorie des Vāmana (ca. $800 \mathrm{n}$. Chr.): Der Stil ist geregelte Wortfügung; das regelnde Prinzip sind die Stiltugenden; die Lehre von den 'Zuständen' (Ausdrucksbewegung, Gefühl, Stimmung) im Drama. Zum Grund- und Eckstein eines Systems wurde die Stimmung aber erst in der uns vollständig erhaltenen Schrift eines unbekannten Autors aus der 1. Hälfte des 9. n. Chr. vom 'Ton' (dhvãni), deren Ziel ist, 'die Herzen der 
Leute von Geschmack zu erfreuen'. Der Eindruck der Abhandlung ist ein günstiger und fast allseitig befriedigender: ruhige, sachliche und scharfsinnige Fassung des Problems; wie in der ind. Poesie überhaupt, so auch hier Bevorzugung der erotischen als der besonders 'erforderlichen' Stimmung, im Gegensatz wozu auffällige Vernachlässigung des eigentlich Tragischen, das überhaupt in der ind. Dichtung nie so recht herausgebildet worden ist. Vielfach moderne Theorien: 'unausgesprochener Inhalt', 'Idee' eines Kunstwerkes, eine Art Ästhetik und Psychologie des 'UnbewuBten'; das bloBe Gefallen am Inhalt eines Gedichtes ist eine 'unselbstische Freude'. Aus der Fülle technischer Vorschriften sei nur das Prinzip der 'Steigerung' hervorgehoben. Die Theorie soll Respekt haben vor der bestehenden Kunst: allerdings werden nur 6 Klassiker anerkannt, daranter Kālidāsa. Voraussetzung unserer Poetik ist, daß̧ der Dichter in Stimmung sein muß, wie ja auch Schiller und Tolstoi die Erweckung einer Stimmung als Hauptzweck der Kunst ansehen, und wie auch Zola die Wirklichkeit durch ein Temperament betrachtet wissen will. - Aus unserem Werke geht jedenfalls hervor, daß die ind. Ästhetik ganz wie die Aristotelische empirischer Natur ist, und daß die empiristișche Ästhetik die ursprüngliche Anschauungsform der ästhetischen Theorie ist. Mögen auch die Ausführungen des unbekannten Verfassers über die Arten des Poetischen lückenhaft sein, so sind sie doch nicht weniger wertvoll.

\section{Literaturgeschichte.}

14. Frazer R. W. A literary history of India. 2 nd. impr. London, Unwin 1907. 486 S. 12 s. 6 d.

15. Reed E. A. Hindu literature; or, the ancient books of India. New issue. Chicago, Scott, Foresman \& Co. 1907. XVIII, 410 S. 1.50 Doll.

16. Oertel H. Contributions from the Jāiminiya Brāhmana to the history of the Brāhmana literature. JAOS. 28, 81-98.

Sixth series: The story ot Uśanas Kāvya, the three-headed Gandharvan, and Indra : Einleitung, Text, Übersetzung mit Anmerkungen und Erklärung des Sprichwortes: 'Die Erde hat Ohren' sowie des Motives von dem durch die Frau verratenen Geheimnisse des Gatten.

17. Schrader O. Bibliography of Sankhya-Yoga-Samuccaya works. Adyar, Theosophist Office. 1906. $18 \mathrm{~S}$.

18. Franke R. O. Dīpavaṃsa und Mahāvamsa. WZKM. 21, 203-250; 317-352.

Bezieht sich auf die Geigers Buche: "Dīpavamsa und Mahāvamsa und die geschichtliche Utberlieferung in Ceylon" zugrunde liegende, bereits von Oldenberg in der Einleitung zu einer Dipavamsa-Ausgabe im Wesentlichen aufgestellte Theorie, der Dipav. sei nicht die Quelle des Mahāv., sondern beide Werke, und mit ihnen z. B. die geschichtl. Einleitung von Buddhaghosas Samanta-Pāsādikā beruhten auf einer allen gemeinsamen Quelle, nämlich auf einem besonderen, historischen $\mathrm{Ab}$ schnitte der alten, uns verlorenen singhalesischen Ațthakathā zum PāliKanon. - Kap. 1. Kanonische Gāthās und Gāthā-Stủcke im Dīpavamsa. - Kap. 2. Folgerungen aus den kanonischen und Dipavamsa-Parallelen (es existiert vorläufig kein Grund, die Berechtigung der eigenen Worte des Dipav. -Verfassers zu bezweifeln, der sein Werk als 'ohne Vorgănger und ohne Seinesgleichen' bezeichnet). - Kap. 3. Wiederholt vorkommende 
Strophen und Strophenteile im Dipavamsa. - Kap. 4. Gemeinsame Strophen und Strophenteile im Dīpavamsa und Mahāvamsa. - Kap. o. Kongruenzen der Samantapāsădika-Einleitung und des Dipavamsa. -

19. Hüttemann W. Die Jñāta-Erzählungen im sechsten Anga des Kanons der Jinisten. (Diss.). Straßburg, Trübner 1907. VII, $49 \mathrm{~S} .1 .50 \mathrm{M}$.

Bespr.: Oldenberg, H., Deutsche Literaturztg. 1907, 1563 f.; Hertel, Literar. Zentralbl. 1907, 1180 f.; Lévi, S., Rev. crit. 1907, II, 83 f.

\section{Grammatik. Lexikographie.}

20. Bartholomae Chr. Arica. IF. 21, 347-354.

21. - Zur Gerundivbildung im Arischen. KZ. 41, 319-335.

Erklärung der im Indischen und Iranischen sich findenden Gerundiva, ihrer Abweichungen und Bedeutungen.

22. - Zu den arischen Wörtern für 'der erste' und 'der zweite'. IF. 22, $95-116$.

23. Denison T. St. Nauatl or Mexican, in Aryan phonology (not including formative syllables). Chicago, Denison 1907. III, $24.75 \mathrm{c}$.

24. Wackernagel J. Indisches und Italisches. KZ. 41, 305-319.

\section{4. kuibera-}

1. Ahd. bibēn : ai. bibhéti. — 2. Ai. áduhat. - 3. Pāli gijjha-. -

25. Ribezzo F. Die Deminutiva der altindischen Sprache und ihre indogermanische Herkunft: ein am Indogermanischen Institut zu Leipzig gehaltener Vortrag. Neapel, Tessitone (Leipzig, Harrassowitz) 1907. $41 \mathrm{~S} . \quad 0.80 \mathrm{M}$.

26. Thurmeysen R. Altindisch étavdi. Mélanges de linguistique offerts à Ferd. de Saussure. S. 223-227.

27. Keith A. B. The use of the 'passive gerund' in Sanskrit. JRAS. 1907. S. $164-166$.

Mit Bezug auf des Verfassers Artikel über diesen Gegenstand (JRAS. 1906. S. 693) und Rouses Bemerkungen hierzu (JRAS. 1906. S. 992). "The history of the use renders it certain that the real rendering as felt by the users is ..., taking the word as an impersonal passive, the passive sense being in either case retained, just as $I$ hold, that the active sense of the Sanskrit gerund is never lost."

28. Andersen D. The Sanskrit word for 'bow' (i. e. fiddlestick). Int. Musikgesellschaft, Basler Kongreb. 1906. S. $244 \mathrm{f}$.

29. Kern H. Vaitulya, Vetulla, Vetulyaka. Versl. en Meded. Ak. Wet. Amst, Afd. Letterk., 4. R. 8, 312-319.

30. Grierson G. A. Vëthadipa. JRAS. 1907. S. 166.

Bemerkung zu Fleet (The tradition about the corporeal relics of Buddha: JRAS. 1906. S. 655 ff. $881 \mathrm{ff}$ ), dab die von diesem vorgeschlagene Gleichsetzung Vethadipa-Bettiah (auch Bettia, Bettiā im heutigen Champāran-Distrikt) nicht richtig sein kann wegen des dentalen $t$ in betiyā.

31. Leamann E. und J. Etymologisches Wörterbuch der Sanskrit-Sprache. 1.Lfrg. Einleitung und a-jū. (=Indica. Texte, Übersetzungen und Studien ans den Gebieten der ind. Religions-, Kultur- und Sprachgeschichte, in zwanglosen Heften hrsg. von E. Leumann. Heft 1). Leipzig, Harrassowitz 1907. $112 \mathrm{~S}$. 80. $2 \mathrm{M}$.

Beepr.: Hertel, Literar. Zentralbl. 1907. S. 991-903. 
Texte und Untersuchungen darüber.

32. Oldenberg $\mathrm{H}$. Vedische Untersuchungen. ZDMG. 61, 803-836.

18. Zu den Verbalpräfixen (im Hauptsatze steht das Präfix normalerweise an der Spitze des Satzes; im Nebensatze herrscht in älterer Zeit die Verbindung von Präfix und Verb keinesfalls in höherem Grade vor als im Hauptsatze; allmähliche Zunahme der Verbindung des Verbs mit 2 und später auch mit 3 Präfixen). 19. Zur Stellung der Vergleichungspartikeln im Rgveda (die Voranstellung von $i v a$ und $n \dot{\alpha}$ ist, wenn überhaupt vorhanden, von ganz untergeordneter Bedeutung; einige andere Fälle abnormer Stellung beider Partikeln). 20. Die enklitischen Formen des Pronominalstamms $a$ - (gegen Delbrück, daß bei adjekt. Gebrauch diese Formen, immer deiktisch stehend, betont seien: auch adjekt. Verwendung enklit. Pronominalformen). 21. Scheinbar einsilbiges iva im Rgveda (verwirft eine Nebenform $v a$ für $i v a$ ). 22. $s a ̊$ und sáh im Rgveda (der Gebrauch des Rgveda nähert sich der späteren Regel: sá vor Konsonant, sáh vor Vokal). 23. Zweisilbige Aussprache des $r$ (Sammlung der in Betracht zu ziehenden Stellen: sichere Entscheidung nicht zu treffen).

33. Scheftelowitz J. Zur Textkritik und Lautlehre des Rgveda. WZKM. $21,85-142$.

Zugrunde gelegt ist das kaśmīrische RV-Ms. (K-RV). "Inhaltsangabe: Varianten des K-ṚV. Die Behandlung des ursprünglich auslaut. $s$. Die Gesetze, nach denen eine ursprüngl. Geminata im Wortinnern vereinfacht wird. Inlaut. ss wird nie $\mathrm{zu}$ ts. Die Bedingungen, unter denen ein auslautender Konsonant vor einem gleichlautenden anlautenden Konsonanten vereinfacht wird. Die Bedingungen, unter denen ein Konsonant verdoppelt wird. Reduktion gewisser Konsonantengruppen. Eigentümliche Behandlung der Nasale im Wort $-u$. Satzsandhi. Die Bedingungen, unter denen anlaut. $c h$ zu $c c h$ wird. $m, n$ im Versauslaut. aiy zu aiyy. Eigentümliche Behandlung des $r$. Die Bedingungen, unter denen ein Wechsel der Zischlaute im K-RV stattfindet. Bereits in vedischer Zeit ist $\boldsymbol{s}$ in der Aussprache schwer von $s$ zu unterscheiden gewesen. $s+s$ ist lautgesetzlich nie zu $k s$ geworden. In RV keśin, kóśa, sákrt, śru-, ścañc, śvātra, śubh, sact, śávas, śvasiti, śusati geht $s$ nicht auf ursprüngl. $s$, sondern auf idg. $\hat{k}$ zurück. Die Beispiele, in denen urind. $\dot{s}, s$ zu ved. $s$ geworden

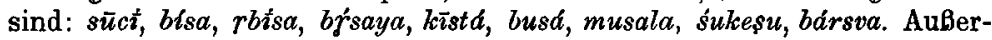
halb des RV gehen die drei Zischlaute häufig regellos durcheinander. Übergang von $v$ zu $b$. Abweichungen des K-RV im Sandhi. Dialektische Spuren im K-RQV-Manuskript. Die Arten der Schreibfehler im K-ḄV."

34. Garnier K. v. Die Präposition als sinnverstärkendes Präfix im Rigveda, in den Homerischen Gedichten und in den Lustspielen des Plautus und Terenz. Diss. Heidelberg. Leipzig, Druck v. G. Kreysing 1906. VI, 64. 35. Hopkins E. W. Aspects of the Vedic dative. JAOS. 28, 360-406.

1. Dative and Genitive after bhū and ásti. 2. Ūtí and other forms with bhū. 3. The gerundives havya and idya with the dative. 4. The syntactical construction of verbs of speaking in RV. and AV. 5. The dative in the Upanishads. 6. Verbs of Speaking in the Upanishads.

36. Neisser W. Zu Rgveda V, 61, 12. ZDMG. 61, 138.

Wendet sich gegen die Dumont-Brunesche Übersetzung dieses Verses als nach verschiedenen Seiten hin bedenklich, glaubt die Schwierigkeit einwandfrei lösen zu können durch Annahme von yéșām im Sinne von 
yá (d. i. yé) eșām und übersetzt: 'Die (sc. die Maruts) in ihrer Schönheit über die Welten hinstrahlen auf ihren Wegen, wie die goldene Scheibe droben am Himmel".

37. Sohroeder L. v. Vimanyu RV 1, 25, \& 'Zorn stillend', 'Grimm vertreibend'. WZKM. 21, 314-316.

Im Gegensatze zu den Interpretationen von Roth, Graßmann, Benfey, Ludwig nimmt v. Schr. das Wort in dem von Sāyaṇa gefaBten Sinne (ein Kompositum von vi mit manyu), in dem es auch im Kamāras. und Bhãg. Pur. vorkommt. Die Präposition $v i$ hat in Kompositis öfters eine dem $a$ oder an entsprechende Bedeutung. Sprachlich liegt hierzu keine Schwierigkeit vor.

38. Sen A. C. The religion of the Rigveda. J. of the Ind. Res. Soc. 1, I-IV.

39. - The hero-gods of the Rigveda. J. of the Ind. Res. Soc. 1, 1-38.

1. The pitris or the Vedic fathers. 2. King Vivasvān.

40. Satyavrata Sámasrami. Niruktálochanam. (A guide to Yáska's Nirukta.) Calcutta, Hitavrata Sámakantha 1907. 290 S. 2 Rs. 8 a.

41. Svámi Vishveshvaránand a. Svámi Nityánand. A complete alphabetical index of the words occurring in the Atharvaveda. Bombay, Nirnaya Ságara Press 1907. 269 S. 2 Rs.

42. Windisch E. Zu Kaușitakibrãhmaṇa Upanișad I, 2. Berichte Sächs. Ges. d. Wiss. Philol.-hist. Kl. 59, 111-128.

Hinweis auf zwei alte Versionen des Jaiminiya Brāhmaña zu der Darstellung des Schicksals der Seele nach dem Tode im Anfang der Kaușītaki-Brāhmaṇa-Upanișad, "die zwar auch noch nicht alle Schwierigkeiten erledigen, aber uns doch in einigen Punkten noch über Böhtlingk und Deussen hinaus konimen lassen". Zuerst folgen die beiden Textstellen, die dann unter Zuhilfenahme philologischer Kritik mit der K.-Br.-Up. in Parallele gesetzt werder. Nur einiges sei andeutungsweise aus dem reichen Inhalte angeführt. In der K.-Br.-Up. wird die Lehre von den Schicksalen der Seele nach dem Tode auf einen bestimmten Brāhmanen zurückgeführt, im J.-Br. erscheint sie dagegen als brāhmaṇa-artige Betrachtung im AnschluB an die Totenfeiern. In den Einzelheiten der Wanderung der Gestorbenen weichen die verschiedenen Überlieferungen voneinander $a b$, im Endresultat stimmen sie aber überein, indem die Seelen sämtlich in den Mond gehen, der durch die als Lebensodem der Menschen gedachten Seelen in der 1. Hälfte anschwillt, während er sie in der 2. Hälfte zu neuen Geburten bringt. Auch sonst zeigt der Mond vielfach offenbare Beziehungen zur Menschenwelt. Bemerkenswert ist in der Darstellung des J.-Br., daß die Welt des Brahmā für die wandernde Seele nicht vorhanden, daß die Welt des Āditya und des Mondes für sie das Ende ist, offenbar einer älteren vedischen Anschauung entsprechend. Aber der Gedanke ist hier schon aufgekommen, daß die Seele aus der Himmelswelt wieder zur Erde gelangen kann. Doch scheint das hier noch in das Belieben der Seele gestellt zu sein, wogegen nach der späteren brāhman. und buddhist. Lehre die Seele vom Himmel fällt, wenn ihre Zeit um ist. Eigenartig sind die visionären Phantasien in bezug auf die verschiedenen Stationen, die die Seele bei der Rückkehr zur Erde durchmachen mu日, und auf denen allen sie die zum Leben notwendigen geistigen Eigenschaften und körperlichen Bestandteile erhält, um schlie日lich als neue vollständige Persönlichkeit wieder auf der Erde zu erscheinen. 
43. Wober A. Eine Betrachtung einiger Lehren der Upanishaden. (=Geheimwissensch. Vorträge. No. 27.) Leipzig, Theosoph. Centralbuchh. 1907. $40 \mathrm{~S} .0,30 \mathrm{M}$.

44. Coco A. Idee e pratiche educative nel Mānavadharmaçāstra. Messina, Trimarchi 1906. $90 \mathrm{~S}$. $2 \mathrm{~L}$.

45. Šerbatskoj F. J. 0 pripisyvaemom Majtrejě socinenii Abhisamayālamkāra (Sur l'Abhisamyālaṃkāra attribué à Maitreya). Izv. Imp. Ak. Naúk (Bulletin de l'Ac. des sc. de St-Pétersbourg) 1907. 6 Sér., 1, 115-117.

46. Hari Charan Sen. Paryáya Muktávali. A collection of pearls of the synonyms of the names of things (used in Ayurvedic science of medicine). 3rd ed. Cuttack, Syámasundar Sáthiyá 1907. 44 S. 8o. 5 a.

47. Hoernle A. F. R. The authorship of the Charaka Samhita. Arch. f. Gesch. d. Med. 1, 29-40.

In Wirklichkeit ist die Ch.-S. nur eine dem 2. Jahrh. v. Chr. angehörige Ausgabe eines von Agniveśa im 6. Jahrh. v. Chr. verfaßten Werkes, das dieser selber wieder aus den Vorlesungen seines Lehrers Ätreya zuzammengestellt hat. Doch hat $\mathrm{Ch}$. selber seine Arbeit nicht vollendet, sondern ein gewisser Dridhabala aus dem 9. Jahrh. n. Chr. Von den Kommentatoren datiert dann der Gebrauch, das Werk als Ch.-S. zu bezeichnen. Hoernle kommt es in der vorliegenden Abhandlung darauf an, Kaschmir als Heimatland des Driḍhabala zu erweisen und die Übereinstimmung der von Drịh. besorgten Bearbeitung der Ch.-S. mit der überlieferten Kaschmir-Rezension der Ch.-S. festzustellen, und er gelangt dabei zu folgenden 4 gesicherten Ergebnissen: 1. Die Ch.-S. wurde revidiert und vollendet von Dridh. 2. Es gab eine revidierte Rezension der Ch.-S., die als die Kaschmir-Rezension bekannt ist. 3. Dridh. nennt sich selbst einen Einwohner eines Ortes Panchanada. 4. Ein heiliger Platz dieses Namens existierte in Kaschmir. Infolgedessen liegt der Schlub nahe, Drịh. als Kaschmirer anzusprechen und seine Rezension eben mit der KaschmirRezension $\mathrm{zu}$ identifizieren.

48. Roussel A. Les idées religieuses et sociales du Mahābhărata. Ādiparvan. Muséon 8, 343--360.

9. Mort. 'Le poète commence par établir que tous les hommes doivent nécessairement mourir. Ce que nous avons de mieux à faire, en face de cette fatalité inéluctable, c'est de nous résigner et d'en prendre courageusement notre parti'. 10. Destin. \& Le nœud de la Destinée ne peut être défait; rien, dans ce monde, n'est le résultat de nos actes s... 'Les Dieux eux-mêmes ne pouvaient rien contre le Destin; ils le savaient bien'.

49. Mazumdar C. Phallus-worship in the Mahābhārata. JRAS. 1907. 337-339. Samhitā'.

'Phallus-worship was unknown to the compilers of the Mahābhārata

50. Besant A. Winke zum Studium der Bhagavad Gītā. 4 Vortrăge. Übers. v. Helene Lübke. Leipzig, Altmann 1907. III, $97 \mathrm{~S}$. 1,60 M.

51. Hertel J. Zu Hemacandra's Parișișțaparvan II, 446 ff. ZDMG. 61, $497-500$.

Einige Verbesserungen zu Jacobis Ausgabe von Hemacandras Parisiștaparvan. Verfasser pflichtet der Ansicht Jacobis vollständig bei, daß Haribhadra nicht als unmittelbare Quelle Hemacandras zu gelten hat. 
52. - Über einen südlichen textus amplior des Paîcatantra. ZDMG. $61,18-72$.

(Fortsetzung.) Eine Anzahl Erzählungen der Handschrift in deutscher Übersetzung. Zum Schluß Parallelstellen und Nachträge.

53. Zachariae Th. Bericht über eine Handschrift des Hitopadesa. ZDMG. $61,342-355$.

Eine bengalische Handschrift zweifellos jüngeren Datums, aus 85 Blättern bestehend; der Text ist so gut wie vollständig und leidlich korrekt, aber mit keiner der bekannten Handschriften oder Ausgaben des Hitopadeśa übereinstimmend; neben Erweiterungen des ursprüngl. Textes (scheinbar aus dem Pañcatantra entlehnt) finden sich auch Kürzungen. Die Lesarten der Hs. decken sich zuweilen auffällig genau mit denen der ältesten Hs. der Hitopadeśa. Es folgt eine Auswahl der wichtigeren Abweichungen von der Schlegelschen Ausgabe (Bonnae 1829).

54. Hara Prasád Sástrí. A dissertation on Kálidása's Málavikágnimitra. Calcutta, R. Datta 1907. 18 S. 80.

\section{Dialekte.}

55. Wickremaginghe M. de Zilva. Index of all the Prakrit words occurring in "Pischel's Grammatik der Prakritsprachen". Ind. Antiq. Appendix. 36, Appendix (121-132).

56. Duroiselle G. A practical grammar of the Pali language. Rangoon, British Burma Press (London, Luzac) 1907. 346 S. 5 R; 8s. 6.

57. - Pāli reader. I. Rangoon, British Burma Press 1907. 128S. 1 Rs. 8 a.

58. Andersen D. A Pâli reader, with notes and glossary. P. II: A Pāli glossary including the words of the Palli reader and of the Dhammapada (second half). Copenhagen, Gyldendal (Leipzig, Harrassowitz in Kom.) 1907. VIII u. $113-288 \mathrm{~S}$. $6,50 \mathrm{Kr}$. $=7,25 \mathrm{M}$.

59. Johansson K. F. Pali-Miscellen. Le Monde oriental 1907/1908. S. $85-108$.

1. Pal. pahams-. - 2. Pal. pacchi- 'korb". - 3. Das pron. na. 4. Pal. daka- 'wasser'. - 5. Pal. tiracchāna-. - 6. Pal. avocāsi 'sagte'. 7. Pal. sāta-asāta-. - 8. Pal. avīcī 'eine hölle'. - 9. Pal. tumo 'selbst'. 10. Pal. seleti. - 11. Pal. kolāpa-. - 12. Pal. paveni-. - 13. Pal. dandha-. - 14. Pal. simbali. - 15. Pal., sanskr. challa- 'rinde'. - 16. Pal. kilesogha'die sündenflut'. - 17. Pal. svätana-. - 18. Pal. lesa-. - 19. Pal. harissa vanna-. - 20. Pal. vittinna-.

60. Moore J. H. Metrical Analysis of the Pàli Iti-vuttaka, a Collection of Discourses of Buddha. JAOS. 28, 317--330.

Eine Vergleichung der Pāli-Metren mit denen der Vedas, des Epos' und der späteren klassischen Dichtungen im Hinblick auf Alter und zweifelhafte Lesarten. Zur Vergleichung herangezogen sind sloka, tristubh und jagatī, hauptsächlich der erstere, und zwar sowohl der gebräuchlichere \& füßige śloka, wie auch der seltenere 6 füßige, der mahāpañkti. Vorläufig ist das verarbeitete Material noch zu gering, um für den eigentlichen Zweck der Abhandlung in Betracht zu kommen. Nur so viel läBt sich sagen, daB alle 3 Metren freier als im klassischen Sanskrit behandelt sind. Der śloka hat sich von dem vedischen Typ ziemlich stark entfernt, etwas : weniger von dem epischen. Doch läßt sich weder ein direktes Hervorgehen aus dem 
ved. slok $\alpha$ erweisen, noch auch irgend welche Beziehung mit den späteren klassischen Entwicklungsstadien. Als positives Resultat der Untersuchung hat sich ergeben, dab die 11 und 12 silbigen Metren weniger variieren als der sloka.

\section{Religionsgeschichte. Altertumskunde.}

61. Zirchow L. Der arische Glaube. Lissa, Ebbecke 1907. 55 S. 1 M.

62. Lala Gokal Chand. The message of the Vedás. Lahore, Anglo Sanskrit Press 1906. 46, 248 S. 80. 1 R. 4 a.

63. Keith A. B. Some modern theories of religion and the Veda. JRAS. 1907. S. 929-949.

Bespr.: Turchi, N., Riv. stor.-cr. d. se. teol. $3,955$.

Über die Möglichkeit des Vorkommens sonst bezeugter religiöser Gebräuche und Vorstellungen imRigveda : so des Totemismus' (der praktisch gebunden sein könnte an Namen wie die Matsyas, Ajas, Sigrus, Vatsas, Sunakas, Gotamas usw.; auch die Fälle von Theriomorphismus der Götter lieben sich in diesem Sinne deuten; vor vollständiger Sammlung des einschlägigen Materials ist jedoch das entscheidende Wort nicht zu sprechen) und des Menschenopfers (auch diese Frage ist noch nicht zweifelsfrei gelöst; die Tatsache der äuBerst seltenen Erwähnung in der Literatur dürfte wohl eine ganz geringe Praxis dieses Opfers erweisen, wenn auch sein Vorkommen bei den indischen Ureinwohnern die Bekanntschaft der vedischen Inder mit ihm voraussetzen läßt).

64. Bhaves Chandra Banerji. Somes notes on the Vedic sacrifices. J. a. Proc.

ASB. 3, 199-204.

General remarks: Zweck des Opfers bei den vedischen Ariern war 1. Erlangung eines glücklichen Jenseits, 2. - und zwar in weit höherem $\mathrm{MaBe}$ noch - Überfluß an Lebensmitteln und Vieh, ununterbrochene Nachkommenschaft und Sieg über alle Feinde und Nebenbuhler. - Classification: Je nach der Opfermaterie war das Opfer 1. ein Haviryajña oder Getreideopfer, 2. ein Pākayajña oder Speiseopfer, 3. Somayajña oder Somaopfer. - The possible priority of the Soma sacrifices to the corn ones: Diese Altertümlichkeit wird bewiesen 1. durch eine Stelle im Cathapatha Brāhmaña, 2. durch die Präponderanz des Somaopfers in den allgemein für die ältesten angesehenen Hymnen des RV. - Who are entitled to the sacrificial observances?: Die 3 obersten Kasten, jedoch mit der Einschränkung, daß gewisse Opfer nur der einen oder anderen dieser 3 Kasten reserviert sind. Eine Frau kann ein Opfer nur in Gemeinschaft mit dem Gatten darbringen. - An account of the priests in the vedic ceremonials: Man muß unterscheiden zwischen dem darbringenden Priester (dem Brāhmana Ritvik), dem eigentlichen Opferer und den Opfergehilfen, die weder Opferer noch Priester sind. Die gröbte Zahl der bei einem Opfer beschäftigten Priester ist 16, die aber zusammen nur beim sattra (einer großen Somafeier von mehr als 12 Tagen) vorkommen.

65. Thomas F. W. Abhișeka. Encycl. of rel. a. ethics 1, 20-24.

Das Wort bedeutet Besprengung, Weihung durch Besprengung mit Wasser (namentlich zum Königtum) und bezeichnet eine altindische Zeremonie, die im Atharva-Veda öfters, aber nicht im Rig-Veda und SãmaVeda erwähnt wird. Im Yajur-Veda, in verschiedenen Brāhmanas und im Śrautaritual aller 4 Veden findet sich das Wort abhisecaniya als Name 
eines mit dem rījasūya in Verbindung stehenden Ritus'. Der Verfasser beschreibt den Hergang dieser Zeremonie (Personen, Ritual, Veranlassung, Zeit, verwendete Stoffe) und fügt einen Bericht bei über die brahmanischen Vorschriften, die sich auf abhișeka, vājapeya und rājasūya beziehen, und über das dazu gehörige Ritual. Eine Literaturzusammenstellung schliebt den Artikel.

66. Rumball E. A. Sin in the Upanishads. Open Court 21, 609-614.

Der Begriff der Sünde in den Up. ist von dem christlichen wesentlich verschieden. Die Lehre von ihr entspringt keinem einheitlichen Vorstellungskreis, sondern ist das Produkt verschiedener Köpfe und Zeiten. Auffällig ist die verhältnismä日ig geringe Betonung der Sünde, was aber damit zusammenhängt, daß die Up. den Sinn weniger auf die negativen Unrealitäten des Lebens, als auf das einzig wirklich vorhanden Seiende, das Brahman, lenken. Während das Ghristentum die Sünde mehr als die Folge dämonischer Wirkungen betrachtet, inhäriert nach der Lehre der Up. die Sünde dem menschlichen Körper selbst. Diese Vorstellung beruht auf der Identifizierung der bösen Taten eines Menschen mit seiner physischen Natur. Durch sie gelangte man einerseits zu einer gesteigerten Askese, andererseits dazu, in der Befreiung von dem Körper die Befreiung von allem Übel selbst zu sehen. Wie für den Orient überhaupt, so ist auch für die Up. die Verwerfung des 'Begehrens (nach irdischen Dingen)' charakteristisch. Die Befreiung von der Sünde durch gute Werke nimmt auch in den Up. einen gewissen Platz ein, viel wichtiger ist hierfür jedoch die 'wahre Erkenntnis'. Nur wer das Brahman richtig erkennt, hat das ewige Iseben.

67. Lomax M. The meaning of māyā. Theosoph. Rev. 40, 306-316.

68. Hillebrandt A. Indische Sādhus. Nord und Süd 122, 298-310.

Die ganze Einrichtung der Sādhus, der indischen religiösen Bettler, der Fakire der Mohammedaner, ist ein Teil nationalindischen Wesens: nicht bloß eine öffentliche Institution, sondern in gewissem Sinne eine staatsrechtliche Erscheinung, deren Verherrlichung durch die ganze indische Literatur sich zieht, und mit der sich auch die indischen Gesetzbücher bereits in den ältesten Zeiten befassen. Ihre Gelübde, oft mit Selbstquälereien schwerster Art verbunden, umgeben sie in den Augen der Menge mit dem Scheine der Heiligkeit. Wenn auch Buddha die Askese als wertlos verworfen hat, so hat er doch ihre Macht nicht brechen können, eine Macht, die sogar die Götter in Furcht versetzte. Die Askese ist dem Inder ein Mittel zur Kontemplation und Meditation, ihn von irdischem Tun und Denken abzuziehen. Wie man in dem einzelnen Mönche der christlichen Kirche immer den Träger des geistlichen Gewandes ehrt, so auch in Indien in dem Sādhu stets den frommen Diener seines Gottes. Die Sādhus, deren Zahl auf gegen 5 Millionen geschätzt wird, rekrutieren sich aus allen möglichen Sekten und tragen als äußere Merkmale dementsprechende Kennzeichen. Wie vor Jahrtausenden ist auch heute noch der dem weltlichen Leben entsagende Asket das Ideal des Hindutums.

69. La Vallée Poussin L. de. Religions de l'Inde. Bouddhisme. Rev. d'hist. et de litt. rel. 12, 370-386.

"Dans la première partie de cet article, le lecteur trouvera une rapide description du Bouddhisme et de son histoire interne; dans la seconde, qui paraîtra prochainement, la bibliographie des sources et des 
ouvrages européens". 1. Éléments payens du Bouddhisme (keine Reaktion und Reformation in bezug auf die vorhandenen Zustände). - 2. Le concept du Bouddha. A. Le Bouddha, être surnaturel et dieu. B. Çākyamuni, fondateur d'une secte religieuse (. . Le Bouddha historique est, par définition, le fondateur d'une de ces sociétés religieuses [es hat nämlich bereits vor Buddha religiöse Sekten außerhalb der Upanischaden gegeben und Leute, die von sich sagten, daß sie ein Buddha seien, d. h., daß sie erleuchtet seien und den wahren Pfad der Erlösung besäßen], l'inventeur d'un nouveau chemin vers la délivrance. Il affirme posséder la bodhi ou illumination, c'est-à-dire, la vérité grâce à laquelle on sort du cercle des naissances, grâce à laquelle on obtient la délivrance de la pensée, le nirvānaa. Lui-même, quand sa vie terrestre sera arrivée au terme, ne renaîtra plus ...). - 3. Systèmes philosophiques du Bouddhisme, Agnosticisme primitif, Système des skandhas [Petit Véhicule], Systèmes Madhyamaka et Vijñānavādin [Grand Véhicule]. - 4. Le Bouddhisme comme religion, Petit Véhicule, Grand Véhicule, Véhicule tantrique (. . Le panthéon, jamais fermé, jadis hiérarchisé dans un sens spirituel et vraiment bouddhique, est entièrement renouvelé, non seulement par l'intronisation de nouveaux dieux, mais encore pas l'identification des Bouddhas anx divinités çivaïtes).

70. Milloué L. de. Bouddhisme. (Annales du Musée Guimet. Bibl. de vulgarisation. T. 22). Paris, Leroux 1907. 208 S. 3,50 Fr.

71. Carus P. The Dharma or the religion of enlightenment. An exposition of Buddhism. $5^{\text {th }}$ ed., rev. a. enl. Chicago, Open Court Publ. Co. 1907. VI u. 169 S. ; 1 T. 25 c.

72. Suzuki D. T. Outlines of Mahāyāna Buddhism. London, Luzac 1907. 420 S. 8 s. 6 d.

Bespr.: Luzac's Or. List 18, 165 P.

73. Dubois L. L. Saint Francis and Buddha. Amer. Cathol. Quart. Review $32,193-211$.

74. Oldenberg H. Der Buddhismus und die christliche Liebe. Dt. Rdsch. $134,380-389$.

Eine Prüfung der Grundlagen, auf denen die Maitri-Lehre des Buddhismus' als christliche Liebe hingestellt wird (Maitri kommt her von Mitra, dem indo-iranischen Gotte über Treu und Glauben, später so viel wie 'Freund' bedeutend): 1. Beschreibung der buddhistischen Stimmungen der Freundlichkeit und Friedlichkeit (darnach ist die Maitri keine Ausübung werktätiger christlicher Nächstenliebe, sondern eines passiven friedevollen Wohlwollens). - 2. Stellung der Maitri im Seelenleben der Gemeinde und in der Rangordnung ihrer Ideale (weder steht sie im Mittelpunkte religiösen Interesses, noch liegt der Nachdruck auf der Güte gegen die Wesen). 3. Zurückführung der Maitri auf ihre Gründe und Einordnung in den Zusammenhang verwandter Tatsachen (Schwächung der Kräfte, die sich anderwärts der Liebe im christlichen Sinne widmen konnten, durch den Hang der Inder zum Unpersönlichen und Allgemeinen, durch die bei ihnen an erster Stelle stehende Notwendigkeit geistiger Arbeit, durch ihre Kasteiung und Abtötung alles Irdischen). - Resultat: Die Pflanze christlicher Nächstenliebe wächst im Buddhismus nicht in so idealer, selbstverleugnender Kraft wie im Christentum; der Gnadenstand des Christentums stellt eine höhere Stufe der Entwicklung dar, als wie der Buddhismus sie einnimmt; beide 
Lehren gehören eben doch zwei verschiedenen Welten an und können nicht miteinander identifiziert werden.

75. Abhedhānanda S. Das Wort und das Kreuz im alten Indien. Einzige autoris. deutsche Ausg. (Vedānta-Philosophie. Heft 14). Leipzig, VedāntaVerlag 1907. $23 \mathrm{~S}$. $0,60 \mathrm{M}$.

76. Lippl J. Angeblich Buddhistisches im Neuen Testament. Theol.-prakt. Monatsschrift 17, 651-665; 715-720.

77. [Sāyaṇa]. Sarvadarśan. A digest of all the systems of Hindu philosophy. Poona, Hari Náráyan Apte 1907. 174, 10,13 S. 2 Rs.

78. Desai S. A. A study of the Indian philosophy. London, Luzac 1907. $406 \mathrm{~S} .5 \mathrm{~s}$.

Bespr.: Luzac's Or. List 18, $214 \mathrm{f}$.

79. Suali L. Contributi alla conoscenza della logica e della metafisica indiane. Gi. Soc. as. it. 20, 33-64.

Fortsetzung und Schluß zu GSAI. 19 (1906), 283-369 (italienische Übersetzung des behandelten Sanskrittextes mit ausführlichem Kommentar).

80. Deussen P. Allgemeine Geschichte der Philosophie mit besonderer Berücksichtigung der Religionen. I. Bd., 2. Abtlg. Die Philosophie der Upanishad's. 2. Aufl. Leipzig, Brockhaus 1907. XIV u. $401 \mathrm{~S} .9 \mathrm{M}$.

81. Barnett L. D. Brahma-knowledge. An outline of the philosophy of the Vedanta etc. (Wisdom of the East). London, Murray 1907. 114 S. 2 s.

82. Bosc de Vèze E. Traité de Yoga (philosophie occulte hindoue). Paris, Daragon [1907]. $205 \mathrm{~S} .6 \mathrm{Fr}$.

83. Ráma Prasád. Self culture or the Yoga of Patanjali. Adyar, Theosophist Office 1907. $224 \mathrm{~S} .8^{\circ}$.

84. Marshall J. H. Archaeological exploration in India, 1906-7. JRAS. 1907. S. $993-1011 ; 8 \mathrm{~T}$.

Ein vorläufiger kurzer Bericht über die vom Archaeological Department in Indien veranstalteten Ausgrabungen (wegen der erzielten Resultate dieses Mal besonders wertvoll), betreffend: Kasiā ( 2 umfangreiche Klosteranlagen; eine Anzahl stūpas; ca. 500 Tonsiegel); Sārnāth (eine Anzahl Denkmäler, darunter eines von $95 \mathrm{zu} 90 \mathrm{FuB}$ im Geviert; eine ausgedehnte Klosteranlage; Nachweis einer bedeutenden und weithinreichenden Bildhauerschule in Sārnāth zur Zeit der Guptas); Sahribahlol; Pagān (the 'Pagoda of the curling leaf', von großer Wichtigkeit für die buddhist. Ikonographie, mit Illustrationen zu den Jātakas, wobei einige neue Jātakas zu den bisher bekannten hinzukommen); Amrāvati; Early Caves (in der Präsidentschaft Madras; wahrscheinlich zu der früliesten Geschichte des Buddhismus' in Südindien in Beziehung stehend ; mit einigen Inschriften in alten Brāhmī-Charakteren); Konarak (Fortsetzung der Ausgrabung der 'Schwarzen Pagode': Standbilder von Bāla-Kṛșna, Viṣnu, Sūrya, Śiva, Yamarāja, von Rssis, von einem Ācārya und dem Ganges); Discoveries in Mārwār (2 Monolithen mit Szenen ans dem Kṛ̦ṇa-Kult; eine Säule mit 4 Bildnissen des Gaṇapati; Reste zahlreicher Tempel).

85. Fleet J. F. The inscription on the Piprahwa vase. JRAS.1907. S.105-130.

"And the presence of that form (sabhaginikanam, wegen des $n$ ) distinctly removes the language of our record from the category of the Mãgadhī of the edicts of Aśōka, and marks it as a local Prākrit, - a dialect, indeed, with some of the peculiarities of Māgadhī, but clearly 
separated from Aśōka's Māgadhī ..." Die Übersetzung lautet nach Fleet: "This is a deposit of relics of the brethren of Sukiti, kinsmen of Buddha the Blessed One, with their sisters, with their children and wives".

86. Barth A. L'inscription P du 'Capiteau des Lions' de Mathurā. Compte rendu de l'ac. des inscr. 1907. S. 384-397.

87. Morton R. S. The city of the sacred Bo-tree. Marvelous ruins unearthed in Ceylon. Cent. Magazine 73,946-954 (ill.).

Ein Bericht über die z. Zt. in Anuradhapura (Ceylon) vorgenommenen Ausgrabungen. Bemerkenswerte Funde: Der Jetawanarama, ein riesiger Tempel, erbaut 330 A. D. vom König Maha Sena zur Feier des Widerrufes seiner Häresie; ein im 2. Jahrh. v. Chr. errichteter Dagoba; der Isurumuniya, der älteste Tempel Ceylons, aus dem Ende des 4. Jahrhs. v. Chr.; ein Baldachin aus Stein mit reichem buddhist. Figurenschmuck; verschiedene Mondsteine; der Thuparama, der kleinste, aber künstlerischste der Anuradhapura-Tempel, nicht viel jünger als der Isurumuniya; der Dalada Maligawa, zur Aufnahme des heiligen Buddha-Zahnes bestimmt. Den Schluß des Artikels bildet eine Beschreibung des berühmten Feigenbaumes, der 288 v. Chr. gepflanzt worden ist und somit 2195 Jahre alt ist. Er stammt von dem Baume, unter dem Buddha das Nirvana erlangte. 88. - A buried civilization of Ceylon: Polonnarua and Dambulla. Scribner's Magazine 41, 24-36 (ill.).

89. Gubernatis A. de. Vita e civiltà vedica (confrontate con la vita degli antichi Romani): Cronache della Civiltà elleno-latina. Roma 1906.

90. Rose H. A. Notes on ancient administrative terms and titles in the

Pañjāb. Ind. Antiq. 36, 348-351.

"A study of the old designations of officials and administrative divisions in the Pañjāb", u. zw. auf Grund der Chambā-Inschriften, die im ganzen 35, zum Teil nicht näher zu spezifizierende Beamte und Titel aufführen, von denen sich nur 2 in die moderne Sprache hinübergerettet haben. An die allgemeine Aufzählung schließt sich eine Betrachtung der einzelnen Beamtenkategorien (der Hauptstadtbeamten, der Beamten mit speziellen Funktionen, der obersten Lokalbeamten usw.). (To be continued.)

91. Sarat Chandra Mitra. Arboriculture and horticulture in ancient and mediaeval India. J. Anthr. Soc. Bo. 7, 526-575.

92. Lüders $H$. Eine indische Speiseregel. ZDMG. 61, 641-644.

Bemerkungen literargeschichtl. u. zoolog. Art zu Mahāsutasomajātaka (537), wo die Gewohnheit, Menschenfleisch zu essen, getadelt wird und von den fünfkralligen Tieren, die ein gesetzeskundiger Kṣatriya genießen darf, die Rede ist.

93. Hopkins E. W. The sniff-kiss in ancient India. JAOS. 28, 120-134.

Eine Untersuchung über Art und Weise des Küssens im alten Indien. Wie viele Völkerschaften den modernen (Mund-)Kuß nicht kennen, so hat auch die vedische Literatur kein Wort für 'küssen', sondern nur für 'sniff' (schnüffeln, schnuppern) und für 'smell' (riechen), u. zw. wird der sniffKuß auf den Kopf gedrückt. Sogar der dem Tierreich entlehnte Vergleich des sniff-Kusses mit dem Beschuppern des Jungen findet sich in der vedischen Literatur. Auch nach Auftreten eines Wortes für 'küssen' bleibt das andere noch bestehen. Der sniff-Kuß ist nicht nur ein Ausdruck des Erkennens, des Wiedersehens, sondern auch der Liebe und des Entzückens. 
Im ältesten Dharmaśāstra wird das Küssen als ein 'Trinken der Feuchtigkeit (des Taues) von den Lippen' beschrieben, was im Gesetzbuche des Manu getadelt wird. Das Mahābhārata enthält die Schilderung oines Kusses, der mit dem Kusse der Jetztzeit völlig übereinstimmt. In den Jātakas ist sowohl der Mundkuß, wie auch der sniff-Kuß bekannt, letzterer besonders zwischen Familienmitgliedern. Männer küssen hier einander nur auf Wange, Nacken oder Stirn. Im 3./2. Jahrh. v. Chr. ist der sniff-Kuß noch eng mit dem Ausdruck zärtlicher Gefühlsbewegung verbunden, wie er denn überhaupt von jeher vorherrschend ein Zeichen familiärer Liebe, namentlich zwischen Eltern und Kindern, und inniger, ehrbarer Zuneigung zwischen Freunden ist. Aus den literargeschichtl. Tatsachen ergibt sich, daß bis gegen Ende der vedischen Periode als einzige Art des Küssens nur der sniff-KuB bekannt war. Erst nach diesem taucht der wirkliche $\mathrm{KuB}$ auf, der immer üblicher wird, bis der sniff-KuB sehließlich ganz verschwindet. Allerdings muß zugegeben werden, daß der wirkliche $\mathrm{Ku} ß$ vielleicht schon früher existierte, dann aber aus besonderen Gründen eine Erwähnung seiner in der Literatur nicht stattgefunden hat.

94. Hoernle A. F. R. Studies in the medicine of ancient India. P. I. Osteology or the bones of the human body. Oxford, Clarendon Press (London, Frowde) 1907. XII u. 252 S. (ill.). 8o. 10 s. 6 d.

Bespr.: Athenaeum 1907, II, 772; Globus 92, 387.

I. Introduction: chronological. II. Text-critical: the records. III. Anatomical: identifications. IV. Apparatus criticus. - Index.

95. - Studies in ancient Indian medicine. JRAS. 1907. S. 1-18.

Bespr.: L a u fer, H., Zbl. f. Anthr. 12, 336.

(Fortsetzung zu JRAS. 1906, $283 \mathrm{ff}$.) II. On some obscure anatomical terms (continued). 3. Skandha. - 4. Usniha a, same as skandha. - 5. Prsti

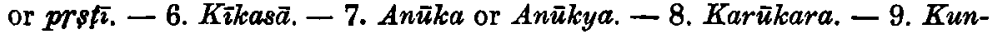
täpa. - 10. Uchlakha, kakā̄tik̄a, kaphoda, kusindha, pratisthā, stana. 11. Bhamsas.

96. Kaviraj Nágendra Náth Sen Gupta. The Ayurvedic system of medicine. Vol. II. III. Calcutta, Kevalrám Chatterji 1907. 723 S. 80. Je 4 Rs. (Leipzig, Harrassowitz. Kompl. $30 \mathrm{M}$.)

97. Thibaut G. Indian astronomy: a historical survey. Indian Thought $1,81-96 ; 193-211 ; 313-334 ; 423-433$.

Wichtiger Überblick über die indische Astronomie. Fortsetzung von Th.s Arbeit im "GrundriB".

98. T. Rāmalingam Pillai. Ãryabhața, or the Newton of Indian astronomyMadras 1905.

99. Sarat Chandra Mitra. Sorcery in ancient, mediaeval and modern India. J. Anthr. Soc. Bo. 7, 332-364.

Leipzig.

E. Schröter.

\section{Iranisch.}

a) Bibliographie.

1. Orientalische Bibliographie, herausgeg. von L. Scherman. 21. Jahrg. (für 1907). Berlin, Reuther \& Reichard 1908/1909. VI, $400 \mathrm{~S}$. $12 \mathrm{M}$.

IV. Indogermanen. 3. Iran. S. 205-214. 
2. Philologiae Novitates, herausgeg. von H. Hungerland. 3. Jahrg. Heidelberg, O. Ficker 1907. $128 \mathrm{~S}$. $4 \mathrm{M}$.

Eranische Sprachen, S. 39-40, 93-94, 125.

3. Orientalistische Lite ratur-Zeitung, herausgeg. von F. E. Peiser. 10. Jahrg. Berlin, Wolf Peiser 1907. VIII, $656 \mathrm{~S}$. $12 \mathrm{M}$.

4. Wilhelm E. Perser. Jahresber. d. Geschichtswissenschaft. 30. Jahrg. 1907. Erste Hälfte. Berlin, Weidmannsche Buchhandlung 1909. S. 65-88.

\section{b) Allgemeines.}

(Geschichte. Kalender. Archäologie.)

5. Christensen A. L'empire des Sassanides, le peuple, l'état, la cour. Mémoires de l'Académie des Sciences et des Lettres de Danemark, Copenhague. $7 \mathrm{me}$ série, Section des Lettres. t. 1, no. 1. Kobenhavn, Bianko Lunos Bogtrukkeri, Hovedkommissionaer Andr. Fred. Høst \& Søn, Kgl. Hof-Boghandel. $120 \mathrm{~S}$. $3,75 \mathrm{Kr}$.

6. Texts, Persian Historical. Volume V. Part II of the Tadhkiratu 'l-Awliyá ('Memoirs of the Saints') of Muhammad Ibn Ibráhím Ferídu'ddín 'Attár. Edited in the original Persian, with preface, indices and variants, and a comparative table showing the parallel passages which occur in the Risálatu 'l-Qushayriyya of Abu 'l-Qásim Al-Qushayrí, by Reynold A. Nicholson 1907. London, Luzac \& Co. Leide, E. J. Brill.

7. Irvine W. Ahmad Shāh, Abdālī, and the Indian Wazīr 'Imād-ul-Mulk (1756-1757). IA. 36. 1907. S. $10 \mathrm{ff}$., $43 \mathrm{ff}$., 55 ff.

Englische Übersetzung eines Berichtes des Ghulām Hasan Samin über den 4. Feldzug des A ḩmad Šăh gegen Indien. Text einiger poetischer Stücke. Ergänzende Noten zur Ghronologie des Feldzugs.

8. Beveridge H. Sultan Khusrau. JRAS. 1907. S. 597-609.

Zur Geschichte des Sultans Xusrau, der als ältester Sohn J̌ahangīr's in Lahore am 14. August 1587 geboren wurde. Text dreier persischer Inschriften (aus dem Xusrau Bār bei Allahabad) mit englischer Übersetzung. 9. Modi J. J. Some interesting portuguese documents. Zartoshti. 4, S. 65-77.

Englische Übersetzung von vier portugiesischen Dokumenten aus den Jahren 1710, 1735, 1736 und 1738, die sich auf die Petitionen der Parsen um die Erlaubnis, 'Towers of Silence' errichten zu dürfen, beziehen. "They show, that two centuries ago, the Parsis were very keen in the matter of their custom of the disposal of the dead. Rather than bury the dead in the places where they lived and were they had no Towers, they preferred carrying the corpses to distant places on three days journey to dispose of them in The Towers there."

10. Sachau E. Drei aramaeische Papyrusurkunden aus Elephantine. Philos. und histor. Abh. der kgl. preuß. Akademie der Wissenschaften aus dem Jahre 1907. Berlin, in Kommission bei Georg Reimer. 40. Mit 1 Tafel. 2,50 M.

Verf. findet in den Texten, die aus der Zeit der Perserherrschaft stammen, drei persische Namen, deren Erklärung er versucht. Waidrang = vayu-dareñgha "an dem Luftgenius Vayu festhaltend, zu Vayu haltend, Vayugenosse" aus vayu- $+{ }^{2}$ dar-, vgl. aw. suptidareñga nach Bartholomae 'Gaugenosse'. Nephajan (vielleicht abgekürzt Nephâ) = napao-yana 'Gunst- 
beweis, Gnadengabe der Gottheit Napdo (vollständig Apãm Napâo). Ramandēn = râmanô-daêna 'dem Gesetz des Genius Raman folgend'. Vayu war vielleicht der Genius des 22. Monatstages, des später als Badh, d. i. Wind, bezeichnet wurde. Der Genius des 10. Monatstages AbAn entspricht vermutlich dem aw. apãm napat. Ram endlich ist der Genius des 21. Monatstages. Erweisen sich die vorgeschlagenen Erklärungen als richtig, dann geben sie einen wertvollen Hinweis auf ein Prinzip der altpersischen Namengebung. 'Il (Zoroastre) regarde les jours du mois comme des dieux', s. Theodorus Bar Kêwânai bei H. Pognon Inscriptions Mandaïtes II, 164.

Bespr.: Lidzbarski, M. DL. 1907, 3160 ff. Wichtig ist die Bemerkung, daß die Sprache der Papyri stark mit kanaanäischen Elementen durchsetzt ist, und dab diese Idiomismen über die aramäische Amtsprache sogar in das religiöse Schrifttum der Perser gedrungen sind.

11. Müller F.W. K. Die 'persischen' Kalenderausdrücke im chinesischen Tripitaka. Sitzungsberichte der kgl. preuß. Akademie der Wissenschaften 1907. 1. S. $458-465$.

Bringt durch Vergleichung des manichäisch-soghdischen Kalenderbruchstückes M. 115 den Beweis, daß die "persischen' Namen der Planeten und Wochentage im chinesischen Tripitaka nicht neupersische, sondern soghdische Worte sind: soghd. mîr (mp. mihr), makh (mp. māh), wunkhan (mp. varhran, arm. LW. vahagn, vahan), tīr (mp.tīr), wurmazt (mp. öhrmazd), nakhîd (mp. a-nāhit), kềwan (mp. kēēân) - chin. mi (mit), mo (mok, mak), yïn-han (wen-hon, un-han), wen-mo-sï (wun-mut-sï), na-cie (na-kit), ci-huan (kai-wun). Veröffentlichung des Kalenderbruchstückes, welches die Tätigkeit der Manichäer als Vermittler zwischen west- und ostasiatischem Wissen beleuchtet. Text nach einer Photographie auf Tafel 6, Umschreibung in lateinischen und arabischen Lettern, Übersetzung, Herstellung folgender Serien: 1. Die Namen der Planeten (soghd.). 2. Die Namen der fünf Elemente (soghd.). 3. Der zehnteilige chinesische Cyklus. 4. Der zwölfteilige Tierkreis (soghd. mit chinesischen und indischen Lehnwörtern). Im Anhang Mitteilung der soghdischen Monatsnamen nach den drei soghdischen Fragmenten M. 148, M. 595, M. 147.

12. Gray L. H. On Certain Persian and Armenian Month-Names as Influenced by the Avesta Calendar. JAOS. 28. 1907. S. 331-344.

Belege für die Ausbreitung des Einflusses des Zoroastrischen Kalenders, der sich nicht nur auf Chorasmia und Sogdiana, sondern auch auf Armenien und Kappadokien erstreckte. 1. Fravartīn, Name des 1. Monats im Jahr (März-April), überall 'Neujahr' aus aw. * nava sarøda genannt: chor. nausärč, soghd. nosard; Name des 19. Monatstages: chor. ravajn, soghd. fravad. 2. Artavahist, Name des 2. Monats: chor. ardvasit, kapp.

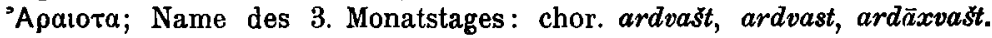
3. Harvatat, Name des 3. Monats: chor. harvadad; Name des 6. Monatstages: chor. harvadād, soghd. radad. 4. Tìr, Name des 4. Monats: chor.

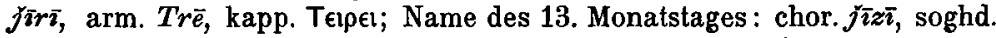

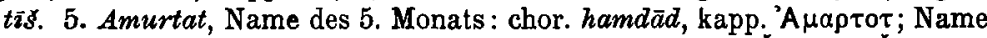
des 6. Monatstages: chor. hamdād, soghd. mardad. 6. Satrvēr, Satrēvar,

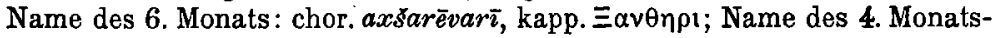
tages: chor. axsarēevari, soghd. xsatšvar. 7. Mitr, Name des 7. Monats: kapp. Mïri, arm. Mehekan. 8. $\bar{A} b \bar{a} n$, Name des 8. Monats: soghd. abānj,

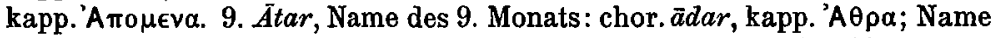
des 9. Monatstages: chor. $\bar{a} d a v$, soghd. $\bar{a} t a s . ~ 10 . ~ D \bar{e} n$, Name des 10. Monats, überall '(Monat des) Schöpfers' (aw. $d a \theta u s \overline{\text { ) }}$ oder 'Ahura Mazda's genannt: 
chor. rēmažd, soghd. xurmažd; Name des 8., 15. und 23. Monatstages: chor.dadav, soghd.dast, kapp. $\Delta a \theta 0 u c \alpha .11$. Vahuman, Name des 11. Monats: chor. asman, arsman, axman, kapp. 'Ocuav. 12. Spandaramat, Name des

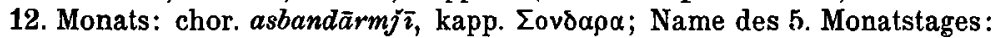
chor. asbandarmǰ $\bar{i}$, soghd. asbandârmad. Es folgt eine Liste der chorasmischen und soghdischen Tagesnamen, die auf dem Einflub des zoroastrischen Kalenders beruhen.

*13. Coorlawalla D. N. The ancient Iranian calendar - as described by Albiruni - with notes and comments. Zartoshti 4. S. 179-215.

14. Hoffmann-Kutschke A. Parsagadae und Persepolis. Philologiae Novitates. 3. Jahrg. S. 7-8.

Parsagadae ist, wie schon Heeren (1. Band, Die Perser, 1824) geäußert hat, die richtige Bezeichnung des Ortes, wo das Kyrosgrab steht. Aus den Trümmern in der Nähe dieses Monuments geht hervor, daß eine große Stadt in nächster Nähe lag. Diese Stadt, bei einigen Autoren kurzweg $\Pi \epsilon \rho c \alpha$ genannt, war das Hauptlager des Stammes der Parsa. Persepolis, heute Tacht-i-Dschemschid genannt, war das Heiligtum der Religion, das abseits der Heerstraße errichtet worden war und zur Abhaltung der großen Kultfeste und der Krönungsfeier diente.

15. Herzfeld E. Pasargadae. Aufnahmen und Untersuchungen zur persischen Archäologie. Berliner Diss. 1907. 32 S. mit einer Karte von Fārs und Kirmän.

I. Teil. Topographisches. Resultat: Die Lage der Ruinen von Mešhed i murghāb, dessen Identifizierung mit Pasargadae von vielen aufs heftigste bestritten wird, stimmt durchaus mit der Vorstellung der Klassiker von der Lage Pasargadaes überein. Man dürfte mit Recht an die Ruinen, welche fünf Mal die Inschrift : "Kyros, der König, der Achaemenide" tragen, mit dem Vorurteile herantreten, in ihnen die Reste der alten Kyrosstadt wiederzufinden. Dieses bestätigt sich nicht nur, sondern gerade aus ihrer Betrachtung entspringt der viel sicherere, weil völlig urkundliche Beweis, dal wir eine Gründung des großen Kyros und also Pasargadae vor uns haben. - II. Teil. Archäologisches wird mit dem I. zusammen in "Klio, Beiträge zur alten Geschichte" erscheinen.

16. Weißbach F.H. Über die babylonischen, assyrischen und altpersischen Gewichte. ZDMG. 61. S. 379-402. 948-950.

17. de Morgan J. Les travaux de la délégation scientifique en Perse au cours de la campagne de 1906-1907: Académie des inscriptions et belles-lettres. Comptes rendus des séances de l'année 1907. S. 397-413.

Bericht über archäologische und epigraphische Funde: 1. Statue du roi Manistusu (face). 2. Statue du roi Maništusu (dos et texte). 3.-6. Céramique susienne.

Mythologie, Religion.

18. Dähnhardt Oskar. Natursagen, eine Sammlung naturdeutender Sagen, Märchen, Fabeln und Legenden. Bd. 1: Sagen zum alten Testament. Leipzig und Berlin, B. G. Teubner 1907.

* Die mit einem * gekennzeichneten Bücher oder Abhandlungen waren dem Referenten, der die Verfassung der iranischen Bibl. plötzlich übernehmen mußte, nicht zugänglich. 
Nachweis fremder Einwirkungen auf die Sagen zum alten Testament. Iranische Einwirkung zeigt die dualistische Schöpfungssage (S.1-38), die Sage vom Teufel und dem Wächterhund (S. 89-111), die Sage vom Raub der Sonne oder Krone (S. 136-142), die Sündflutsage (S. 257-294). 19. Siecke Ernst. Drachenkämpfe. Untersuchungen zur indogermanischen Sagenkunde. Mythologische Bibl., herausg. von der Gesellschaft für vergleichende Mythenforschung. Leipzig, Hinrichsche Buchhandl. 1907. Bd. 1, Heft 1.

S. 21-28. Trita-Thrita bedeutet 'der Dritte', nämlich der Mond am dritten Tage nach der Konjunktion. Diesem Mondgott kommt dieselbe Drachentötung zu wie Indra, und zwar die Tötung des Monddrachen. Traitana-Thraêtaôna-Feridun, Kereçậpa, Zāl und Rustam sind als Abkömmlinge des Trita-Thrita ebenfalls Drachentöter.

20. Camont Franz. Notes de mythologie manichéenne. Revue d'Histoire et de littérature religieuses. 1907. no 2.

*21. v. Schroeder L. Altrarische Religion. ÖRs. 11. II (1907), 110-121.

*22. Henry Victor. Soma et Haoma, le breuvage de l'immortalité dans la mythologie, le culte et la théologie de l'Inde et de la Perse. Bibliothèque de Vulgarisation. Tome 20. Conférences faites au Musée Guimet.

Conférence très intéressante, où il parle de la nature de la plante des montagnes qui fut originairement celle d'où l'on extrayait le breuvage sacré, décrit l'agnistôma, puis le yasna ou sacrifice correspondant chez les Mazdéens. M. V. H. pense qu'il s'agit originairement d'un charme de pluie. Soma est associé à la lune, taureau céleste, qui répand la rosée ou qui gronde dans l'orage. La littérature dogmatique de l'Avesta confirme cette interprétation: "breuvage saint, pluie, fécondité, ambroisie, immortalité, voilà tous les concepts de l'Inde ratifiés et clarifiés par la dogmatique persane”. (RHR. 1907. 55, 259 f.)

23. Meillet M. A. Le dieu indo-iranien Mitra. JA. Dixième série. 1907. 10. S. $143-159$.

Der Name des Gottes ai. mitrá- m., iran. miøra- m., identisch mit mitrá- m. 'Freund', n. 'Freundschaft', aw. misra- m. 'Vertrag' geht auf die Wz. *mei- 'tauschen, einen Kontrakt machen' zurück, vgl. lat. com-mūnis, sl. miv 'Ordnung, Frieden'; *mei- (oder *meie-) , mild, freundlich' ist fern zu halten. Mitra ist nicht der Freund, sondern die Personifikation des Vertrags oder besser seiner mystischen Kraft. Mitrd- und Váruna- (wie die Ādityas überhaupt) sind "personnalités morales". Ihr Auge, das darüber wacht, dab die Verträge geachtet werden, ist die Sonne, die alles sieht; daher die Beziehung zur Sonne und schlieBlich Identifikation bei den Iraniern. Der iran. Miөra straft die Vertragsbrüchigen, weshalb er auch als Helfer im Krieg gilt. Aw. vouru-gaoyaoitis und ved. gavyūtiȟ ist oin altes indo-iran. Epitheton des Gottes, dessen Bedeutung nicht mehr zu bestimmen ist; gdoyñtih hat wohl den Platz bezeichnet, auf dem der Gläubige seine Bitte an einen Gott, speziell an M., richtete. Váruna muß etwas ähnliches wie Mitra bedeutet haben; es gehört wahrscheinlich zu ai. vratám, 'göttliches Gesetz', aw. urvaitis 'Vertrag' von der Wz. uerésprechen'.

24. Khandalewala, Pestonji Dorabji. The Great Magian. Zartoshti 4. S. 1-8.

Über die Bedeutung Zoroasters und seiner Religion. 
25. Reiner, J. Zarathustra. Berlin und Leipzig, H. Seemann Nachfolger 1907. $1 \mathrm{M}$.

*26. Martinengo-Cesaresco, Countess E. The faith of Iran. Contemp. Rev. 9. S. $489-503$.

*27. Mistri, R. H. Zoroaster and Zoroastrianism. Bombay 1907. $218 \mathrm{~S}$. Leipzig, Harrassowitz. 3,50 M.

28. Johannan, A. and Jackson, A. V. W. Some References to Zoroaster and his Religion. JAOS. 28. 1907. S. 183-188.

Englische Übersetzung eines der persischen Texte, die Salemann im Bulletin de l'Académie Impériale des Sciences de St.-Pétersbourg, 9, 417-594 veröffentlicht hat. Dieser Text Siucar-i Alkälsm-i Sab'ah 'Sketches of Seven Countries' enthält eine Legende, die die groben Schneefälle in der Umgebung Ardabils und des Berges Savalān der Wirkung eines Fluches zuschreibt, den Zoroaster lange vorher ausgestoben hatte, sowie wichtige, auf alter Tradition beruhende Angaben ûber das Leben, die Mission und die Lehre Zoroasters.

\section{c) Sprache.}

29. Hoffmann-Kutschke A. Iranisches bei den Griechen. Philologus 66 (NF. 20) 1907. S. 173-191. Nachträge und Verbesserungen S. 320.

Versuch "ein klares Bild zu geben, inwieweit das bei den Griechen genannte iranische Sprachgut richtig - oder besser gesagt - falsch ist, damit endlich in die Kommentare usw. zu Herodot und zur Anabasis die richtigen Ansichten hineinkommen".

\section{Altpersisch.}

(Texte, Textkritik, Syntax.)

30. King, L. W. and Thompson, R. C. The Sculptures and Inscription of Darius the Great on the rock of Behistûn in Persia. A new collation of the Persian, Susian and Babylonian texts, with english translations etc. With Illustr. Printed by order of the trustees. British Museum 1907. LXXX, 233 S.

Preface von E. A. Wallis Budge. Introduction über Lage und Geschichte der Behistûn-Inschrift, über die Notwendigkeit einer neuen Collation und die Arbeiten der Expedition des britischen Museums unter L. W. King und R. C. Thompson. Analyse und Chronologie der Inschrift. Die historische Erzählung. Der Zustand der Inschrift, I-XLV. List of proper names, XLVII-LXXIX. Text of the inscription of Darius on the rock of Behistûn. I. Der persische Text, 1-91. II. Die susische Version, 93-157. III. Die babylonische Version, 159-210. Index, 211-223.

31. Hoffmann-Katschke, A. Zu den Achamanideninschriften. Philologiae Novitates. 3. Jahrg. S. 102-106.

Textkritische Bemerkungen zur Ausgabe der Bagistantexte durch King und Thompson (s. No. 30).

32. Pedersen, H. Neues und Nachträgliches. KZ. 40 (NF. 20). 130-217.

I. Exegetische und syntaktische Fragen. $\$ \S 1-6$ : zum Altiranischen. 129-134. Ap. abis (Bh. 1. 18) 'auf der andern Seite' mit der lokalen Bedeutung des gr. à $\mu$ pic, aw. aibił (V. 3. 24) 'ohne' mit der privativen Bedeutung desselben griechischen Wortes. Parallelen aus dem Slavischen und Irischen für die Bedeutungsentwicklung 'um' - 'bei, auf' - 'auf die 
andre Seite - 'auf der andern Seite' - 'entfernt von, ohne'. Ap. ma.kāhuva nach elamisch mas-ka-um als maskīanhuvō zu lesen, entweder aus dem assyr.

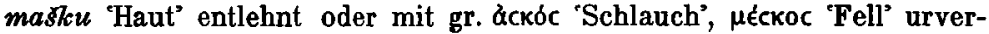
wandt. Ap. appisim parābara (Bh. 1.19) 'es riß ihn (kāram) fort im Wasser'. apisim ist in api (loc.) und sim zu zerlegen; paräbara ist subjektlos.

Awesta.

\section{(Texterklärung.)}

33. Punegar, K. E. Notes on the Tîr Yasht critical and analytical with an introduction on the identity of the star Tishtrya and on the construction and contents of the text. Bombay 1907. Fort printing press.

"The annual phenomenon of the acronycal rising of Tishtrya-Sirius (at the time of the Autumnal Equinox), led our forefathers to name the month, during which Tishtrya was most conspicuous in the firmament, from the star itself. Thus Tishtrya became also the name of a month. But as monsoon set in about the time of the Autumnal Equinox, the month Tishtrya came to be regarded as the first month of the rainy season and the star Tishtrya began to be looked upon as the harbinger of rain. Besides, an angel presiding over rain was created and named Tishtrya". (Introduction VI.)

\section{(Grammatik.)}

34. Wolff, F. Die Infinitive des Indischen und Iranischen. Erster Teil. KZ. 40 (NF. 20) 1-111.

Einleitung: Kriterien für die Grenzbestimmung zwischen Infinitiv und Substantiv und zwischen Infinitiv und finiter Verbalform. I. Abschnitt. Die ablativisch-genetivischen Infinitive: Übersicht. Die ai. Infinitive auf -toh. Die ar. Infinitive auf $-a h$. Im Anhang die iranischen $a h$-Infinitive. Die übrigen ablativisch-genetivischen Infinitive und zwar iranische Infinitive auf $-t \bar{o} i \bar{s}(-t \bar{o} i \underline{t})$ und $\bar{a} t$. II. Abschnitt. Die akkusativischen Infinitive: Übersicht. Die ai. Infinitive auf -tum. Die indo-iranischen Infinitive auf $-(a) m$. Die indo-iranischen Infinitive auf $-\bar{a} m$. Die iranischen Infinitive auf -tim. Im Anhang at-Bildungen.

35. Zubatý, J. Die 'man'-Sätze. KZ. 40 (NF. 20) 478-520.

Inhaltsangabe IF. Anz. 24, 119.

36. Cuny A. [Les préverbes dans le Çatapathabrāhmaṇa.] MSL. 1906/8. 14, 289-331.

État indo-iranien. Préverbes encore complètement libres par rapport à la forme verbale, en indo-iranien. S. 291. 292.

$$
\text { (Etymologien). }
$$

37. Charpentier Jarl. Zur arischen Wortkunde. KZ. 40 (NF. 20), 425-477. Inhaltsangabe vgl. Bibliographie 1906. IF. 24, 117.

38. Uhlenbeck CC. Etymologica. KZ. 40 (NF. 20). 552-561.

39. Marstrander Karl. Etymologische Miszellen. IF. 20, $346 \mathrm{ff}$.

40. Schwyzer E. Nhd. Rahm : jgaw. raoyna-. IF. 21, $180 \mathrm{f}$.

41. Scheftelowitz J. [Zur Textkritik und Lautlehre des Rgveda]. WZKM. 1907. 21, 85-147.

aw. jaidyemi 'verlange, bitte', (air. guidu [! sic] 'bitten', ab. zedati, žęeti 'cupere, sitire", lit. pasi-gendu 'sich sehnen, verlangen'), ai. ghad- 
'lechzen, trachten, gierig sein' in aghattām, aghattam, jighatsati usw. S. 100 f. aw. upa-srvant- 'verletzend, beeinträchtigend', ai. \&ruvat (RV. I.

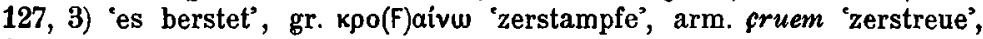
S. 128. aw. kaēs- 'lehren', ai. kīstd 'Sänger', lat. quaero 'zu erfahren suchen, fragen'. S. 131.

42. Holthausen F. Etymologien. IF. 20, 327.

43. Stokes Whitley. Irish Etyma. KZ. 40 (NF. 20.) 243-256.

Aw. cinō (cinah-), cinma (cinman-) 'Liebe, Huld', (ved. cánas 'Gefallen, Befriedigung', canisthas 'sehr gnädig, genehm'), ir. cin 'love, affection, gen. cena aus urkelt. *kenu-. S. $246 \mathrm{f}$.

\section{Sakisch.}

(Namendeutungen.)

44. Hoffmann-Katschke A. Sakisches. Philologiae Novitates. III. Jahrg. S. $64-66$.

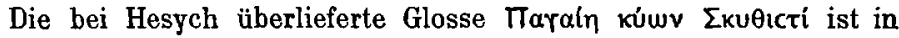

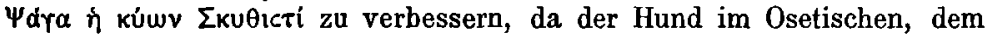
letzten Rest des Skythischen oder Sakischen äfsag (aus $\ddot{a}$-fsaka, mit Umstellung aus sfakka, spaka) heißt und der Volksname Psaccae auch 'Hunde' be-

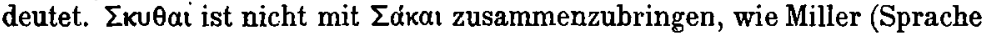
der Oseten, 1903, S. 42) versucht. "Die sonstige Wiedergabe des $\Sigma \kappa u ́ \theta$ l

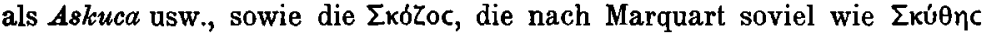
ist, wobei überhaupt $-\theta$ - in orientalischen Namen als -tsch- wiedergebend bereits erkannt ist, lassen eine Form Skutscha erschließen, die die Ur-

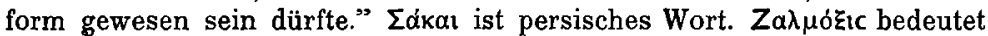

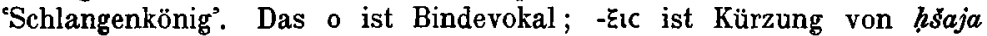

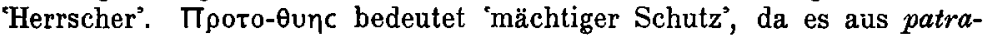
'Wache, Hut' und tua 'stark', ved. tuvi zusammengesetzt ist. Sakisch Dalta ist indo-iranisches Dhatra 'Gesetz'; Orodaltis (Auradalta), der Name des Königs von Bithynien, ist daher mit 'A(h)uras Gesetz habend'

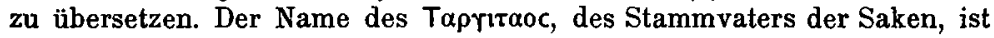

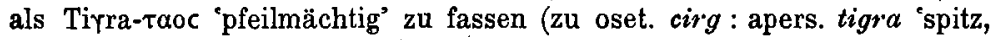
Pfeil' und -Taoc : tuvi 'mächtig').

\section{Mittelpersisch.}

(Texte und Übersetzungen.)

45. Vendidad. Avesta text with Pahlavi Translation and Commentary, and glossarial index edited by Shams-ul-ulma, Sindar, Khan Bahadur, Dastoor Hoshang Jamasp, Parsi High-Priest of the Deccan with the assistance of Mervanji Manekji Gandevia in two volumes. Bombay 1907. Government central book depôt. Vol. I. The texts, 9. 695. Vol. II. Glossarial Index. 7, 271.

Der Text des Vendidad ist so gegeben, wie er in den Handschriften mit der Pahlavi-Übersetzung erscheint. Den einzelnen Paragraphen des Avesta-Textes folgt die Pahlavi-Übersetzung. Erklärende Zusätze sind durch eckige Klammern gekennzeichnet. Es wurden 11 Manuskripte benützt: DN 1836, DJJ 1767, DJE 1825, DJR 1823, DR 1755, MU (31 Folio's) etwa 1323: the handwriting is certainly Mitra-Âwân's, $M_{1}$ etwa 1832, $\mathrm{MU}_{2}$ etwa $1833, \mathrm{MU}_{3}$ etwa 1607 , IM 1585, und die Ausgaben Geldners und Spiegels verglichen. Die Varianten der Handschriften und abweichende 
Lesungen Spiegels (im Pahlavi-Text) sind unter dem Texte angeführt. Ein Appendix 681-693 handelt merkwürdigerweise über die verschiedenen Eulenarten. Der Index enthält alle Pahlavi-Wörter und diejenigen AvestaWörter, die in dem Pahlavi-Kommentar angeführt sind.

46. Dinkard, the. The original Pahlavi text; the same transliterated in Avesta characters; translations of the text in the English and Gujerati languages with annotations and a glossary of select words by Darab Dastur Peshotan Sanjana. Vol. X. Published under the patronage of the Trustees of the Sir Jamshedji Jejeebhai translation fund. Leipzig, Otto Harrassowitz 1907. XXVI. 26. 33. 31. 33. 27. 25. 4. 30. 28. $10 \mathrm{~S}$. $15 \mathrm{M}$.

Contents of the text of book 5 and 6 (p. III -V). Introduction über die Geschichte und die Zusammensetzung des Pahlavi-Dînkard, als dessen Grundlage die Pahlavi-Übersetzung eines gleichnamigen Awesta-Originals angenommen wird (S. VII, VIII), über die benützten Handschriften (S. IX-XIII) und über den Inhalt des 5. und 6. Buchs (S. XIV-XXVI). The Pahlavi-text of book $\tilde{b}$ (p. 1-26). Transliteration in Avestic characters of book 5 (p. 1-31). Transliteration in Avestic characters of book 6 (p. 1-33). English translation of book 5 (p. 1-27). English translation of book 6 (p. 1-25). Contents of the text of book 5 and 6 in Gujerati (p. 1-4). Gujerati translation of book 5 (p. 1-30). Gujerati translation of book 6 (p. 25). Glossary of select words occuring in the Pahlavi-text of book 5 (p. 1-5), of book 6 (p. 6-10).

Mills Lawrence. The Pahlavi texts of Yasna LXV (Sp. LXIV), edited with the collation of all the MSS. ZDMG. 61. 1907. S. 370-378.

47. Jackson A. V. Williams. On a Pahlavi Bowl-Inscription deciphered by the late E. W. West. JAOS. 28. 1907. S. 345-348.

Abdruck eines Briefes, in dem West dem Besitzer einer sassanidischen (?) silbernen Schale die am Rande derselben befindliche Inschrift

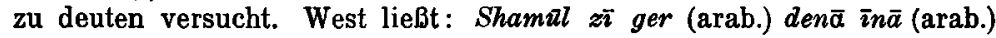
tafshālak mutagad(arab.)-rīsh, val̄̄ zad, zag govbag (govāg?) $\frac{s}{g e r}$. 'Samuel the stranger, pouring out the breakfast of stew of this vessel, consumed it; the witness of it is $\frac{S}{g e r}$. Auf Grund der verschiedenen Schreibung des $s h$, das sowohl in der alten wie in der neueren Form erscheint, schließt West, daß die Inschrift aus derselben Zeit stammt, wie die datierten Münzen der Herrscher von Taparistān, aưf denen sich gleichfalls noch die alte Form des sh neben der neueren findet, also etwa aus der Zeit um das Jahr 710.

48. Mills Lawrence. The Pahlavi texts of Yasna XXII, for the first time critically translated. JRAS. 1907. S. 85-90.

The Pahlavi text of Yasnas LXVI (Sp. LXV) and LXVIII (Sp. LXVII) for the first time critically translated. JRAS. 1907. S. 583-596.

\section{(Grammatik, Etymologien.)}

49. Bartholomae, Ghr. Zur Rechtschreibung des Buchpahlavi: pāhrëxtan oder pahrēxtan? WZKM. 21, 1-10.

Nachweis einer Schreibregel des Buchpahlavi, daß vor $h$ oder $x$ (geschrieben $a$ ), dem $r$ oder $l$ folgt, der kurze Vokal $a$ ( $i$ und $u$ ) plene geschrieben wird. Im Anhang Feststellung der Etymologie von pahl. văhman 
als iran. *ūahumanah- 'der zum guten Sinn gehörige', dessen Bedeutung wie die unseres 'Christenmensch' zu irgend einer, jemand' (np. $b \bar{a} h m \bar{a} n$, bihman) abblabte.

50. - Miran. vinärtan. an. nara- usw. IF. 21, 347-351.

51. - Buchpahl. tasom und apers. $\theta$ r. IF. 22, 101-105.

\section{Soghdisch.}

52. Müller F. W. K. Neutestamentliche Bruchstücke in soghdischer Sprache. Sitzungsberichte der kgl. preuß. Akademie der Wissenschaften. 1907. 1, 260-270.

Vier in syrischer Schrift abgefaßte Handschriftenbruchstücke in soghdischer Sprache, die Texte Galater 3, 25-4, 6 (B 46), Lukas 1, $63-80$ (B 38), Matth. 10, 14 ff. (B. 71 Vorderseite), Joh. 20, 19 ff. (B 71 letzte Seite) enthaltend. Angaben über die Transskription. Zusammenstellung der bemerkenswertesten grammatischen Formen.

53. Salemann C. Manichaeica I. Bulletin de l'Académie impériale des sciences de St.-Pétersbourg. VI. Série. 1907. 1, 175-184.

Vier Handschriftenfragmente aus Urumči mit manichaeischen Schriftzeichen, darunter eines im gewöhnlichen Mittelpersisch der Manichaeer, eines ,sicher "soghdisch"".

\section{Neupersisch. \\ (Texte, Textkritik.)}

54. Beveridge A. S. Further notes on the Babar-Nama MSS. The Elphinstone Codex. JRAS. 1907, S. 131-144.

Beschreibung des in der Advocates' Library gefundenen Elphinstone Codex des Bābar-Nāma oder Vāquỉāt-i-Bābarī, der den tschaghatāischen Text mit zahlreichen türkischen und persischen Noten enthält.

55. Ranking, G. Vorschlag zu einer Textverbesserung im Gulistān. 17. Geschichte. JRAS. 1907, S. 168.

In $y \bar{a}$ ba-tamsvozs (! sic) $u$ russa rā $\underline{d z}$ sav ist statt russa besser rudda zu lesen und zu übersetzen: "either be content to be in perplexity as to your daily bread'.

\section{(Literaturgeschichte.)}

56. Horn, P. Roß und Reiter im Šăhnāme. ZDMG. 61. 837-849.

Wertschätzung der Schilderung von RoB und Reiter und des Verhältnisses beider zu einander als ein Spezimen dessen, was das Šàhnāmo in bezug auf kulturelles Material einerseits und poetischen, mannigfaltigen Ausdruck andrerseits bietet.

57. Shihabuddin Khada Baksh. Maulānā Mu'min Husain of Yazd. ZDMG. 61. 139-141.

Angaben über das Leben und die Rubä’īs eines persischen Mystikers,

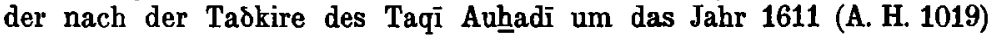
gestorben ist.

58. Phillott, D. C., Lieut.-Colonel. A note on Sign-, Gesture-, Code-, and Secret-language etc., amongst the Persians. Journal and Proceedings of the Asiatic Society of Bengal. New Series, Vol. III, 1907 (Calcutta 1908). p. $619-622$. 
(Grammatik.)

59. Ranking, G. S. A., M. A. University Lectures in Persian. A Primer of Persian. 72 pages. Oxford. Clarendon Press 1907. 2 s. 6 d.

Elemente der Grammatik. Übungsstücke zum Übersetzen aus dem Persischen und in das Persische.

\section{Anhang.}

Mr. J. J. Modi hatte die Güte, dem Referenten auf seine Bitte hin ein Verzeichnis der in Bombay veröffentlichten Bücher und Abhandlungen des Jahres 1907 zuzusenden, aus dem nachzutragen ist: M. B. Davar. Which is the first month of the Zoroastrian Calendar? (in Gujrati.) S. A. Dubash. The Zoroastrian Sanitary Code. - N. R. Kanga. Study of morals in Zoroastrianism (in Gujrati). - J. J. Modi. Lectures and Sermones on Zoroastrian Subjects, Part III (in Gujrati). - J. J. Modi. Episodes from the Shah Nameh. Parts I and II (in Gujrati). - Articles published in the Zartoshti. January 1907. In Gujrati: P. B. Desai. Pouruchista the daughter of the Prophet (Zoroaster). - Kh. E. Pavri. Ancient ceremonies and reforms thereon. - J. J. Modi. An inquiry from Pahlavi, Pazend and other works on the subject of the Number of Days of the Fravardegan. - April and July 1907. In English: D. N. Coorlawalla. The article on Iranian Calendar (s. Nr. 13), continued. N. D. Khandalawalla. Frashaostra, Jamasp and Pouruchista. J. J. Modi. King Salomon's Temple and the Ancient Persians. - J. E. Sanjana. The belief in transmigration, its growth and development in India. - In Gujrati: Kh. E. Pavri. Daremeher or the Court of Meher Davar (i. e. Mithra, the Judge). - P. B. Desai. Kisseh-i-Sanjan or the Parsee Colony of Sanjan. - October 1907 and January 1908 (a joint number of two quarters). In English: J. J. Modi. King Salomon's Temple and the Ancient Persians, continued. - F. W. H. Myens in the "Human Personality and its Survival of Bodily Death" as against the Theory of Reincarnation. (This article contains references to Zoroastrianism.) In Gujrati: P. B. Desai. Kisseh-i-Sanjan, continued. - Kh. E. Pavri. The Astronomy of the Avesta. The Horses and the Chariots of the Yazatas. - J. J. Modi. An inquiry from Pahlavi, Pazend and other works on the subject of the Number of Days of the Fravardegan, continued.

Gießen.

Hans Reichelt.

\section{Armenisch.}

\section{Literatur und Sagen.}

1. Gelehrte Literatur.

1. Acarian H. Die Quellen der Geschichte des hl. Mesrob und der Erfindung der (armenischen) Schriften und Untersuchungen derselben. Paris, Druckerei 'Banaser' 1907. 46 S. 80 ,

2. Akinian N. Gregor, Sohn des Abas, als Verfasser 'des Buchs der Ursachen' (Liber Causarum) [Armen.]. Handēs Amsorya 1907. 21, 132-135.

'Das Buch der Ursachen' (Cod. Arm. Nr. 47 der MechitharistenBibl. in Wien) ist am Anfange des 13. Jahrh. zusammengestellt von Gregor Vardapet, Sohn des Abas ( $f$ zwischen 1217-1221 im Kloster Sanahin in Grobarmenien). 